Cognitive deficit was conceived by Emil Kraepelin (1919) to be a core feature of a single disease, dementia praecox. In contrast, Eugen Bleuler (1923) hypothesized that schizophrenia was the 'common final pathway' for an etiologically mixed group of disorders. The controversy engendered by these two great clinicians survives unscathed in the present diagnostic concepts of schizophrenia. Because schizophrenia is clinically heterogeneous and genetically complex, ICD-10 and DSM-IV diagnoses may not provide the optimal phenotypes for genetic analysis. We developed a novel, endophenotype-based approach to the search for susceptibility genes by generating a composite phenotype that integrates multiple measurements of neurocognition, neurological signs and personality traits using a variant of latent class analysis known as grade of membership. The WA Family Study of Schizophrenia sample (112 families, 388 individuals) yielded two distinct neurocognitive phenotypes, each correlated with schizophrenia: one indexing pervasive neurocognitive deficit and predominantly 'negative' symptoms, and one neurocognitively unimpaired, with florid 'positive' psychotic symptoms. The quantitative neurocognitive trait was used in linkage analysis as a liability covariate, on which each individual in the sample (affected and unaffected) was scored. A 10-cM genome scan, followed by ordered sets analysis resulted in a robust linkage signal (lod score $>3.4$ ) for the neurocognitive deficit subtype on chromosome 6p2522 , whereas the neurocognitively unimpaired subtype was definitively excluded for linkage to the same region. This work supports 'splitting' schizophrenia into genetically distinct subtypes and shows an increase in power resulting from use of composite quantitative endophenotypes.

\section{The Role of Apolipoprotein E in Neuropsychiatric Disorders}

\section{S Sundram}

Mental Health Research Institute, Parkville, Victoria, Australia

\section{Overview}

The most robust genetic predictor of sporadic Alzheimer's disease (AD) is the $\varepsilon 4$ allele of the apolipoprotein E (apoE) gene. This has spawned much interest into the relevance of this lipidbinding protein in this and other neuropsychiatric disorders and the roles it may play in the central nervous system (CNS). This symposium will present work examining the role of the apoE protein in neuronal function and CNS disorders focusing upon $\mathrm{AD}$, schizophrenia and bipolar disorder. Novel data exploring both central and peripheral functions for apoE and its dysregulation will highlight the relevance of this protein in the pathology of these disorders.

\section{9-01}

\section{Role of apolipoprotein E in Alzheimer's disease}

\section{RN Martins}

Centre of Excellence for Alzheimer's Disease Research and Care, Edith Cowan University, Perth, Western Australia, Australia

The $\varepsilon 4$ allele of apolipoprotein E (APOE) is currently the major genetic risk factor for Alzheimer's disease (AD) and is associated with $25 \%-50 \%$ of late-onset AD. Several studies, including our own with the Australian Caucasian population, show that the AD risk associated with the APOE genotype is exerted across most populations worldwide with the exception of the Hispanic and African American populations. However, while the $\varepsilon 4$ allele is generally recognized to play an important role in the pathogenesis of $\mathrm{AD}$, its mechanism of action remains controversial and is poorly understood. Others and we have shown in vitro that one potentially important mechanism of action involves its role in the metabolism of beta amyloid $(A \beta)$ thought to be central to the pathogenesis of $\mathrm{AD}$. We showed the ApoE $\varepsilon 4$ isoform is deficient in binding to $A \beta$ and clearing it from the extracellular space, an event that appears to be through binding of ApoE/A $\beta$ complexes to LRP. Recently, we have shown in vivo that mouse apoE retards clearance from peripheral tissues such as liver and kidney of $A \beta$ injected into the circulation. Furthermore, we show that $A \beta$ administered peripherally does not result in uptake by the brain which is consistent with previous transgenic work for human $A \beta$ showing that peripheral $A \beta$ does not directly contribute to the pool of brain $A \beta$. Taken together with recent findings that proteins that bind $A \beta$ in the periphery enhance its clearance from the brain together with recent findings that proteins that bind $A \beta$ in periphery enhance its clearance from the brain, we propose that human apoE alters $A \beta$ clearance in an isoform-specific manner in vivo such that apoE $\varepsilon 2$ is most efficient and apoE $\varepsilon 4$ is least efficient. 